\title{
Co-solvents as liquid surfactants for boron nitride nanosheet (BNNS) dispersions
}

Touseef Habib, ${ }^{1}$ Dinesh Sundaravadivelu Devarajan, ${ }^{2}$ Fardin Khabaz, ${ }^{2}$ Dorsa Parviz, ${ }^{1}$ Thomas C. Achee, ${ }^{1}$ Rajesh Khare, ${ }^{2 *}$ Micah J. Green ${ }^{1 *}$

${ }^{1}$ Artie McFerrin Department of Chemical Engineering, Texas A\&M University, College Station, TX, USA, 77843

${ }^{2}$ Department of Chemical Engineering, Texas Tech University, Lubbock, TX, USA, 79409

*corresponding authors: micah.green@tamu.edu, rajesh.khare@ttu.edu

\section{Supporting Information}

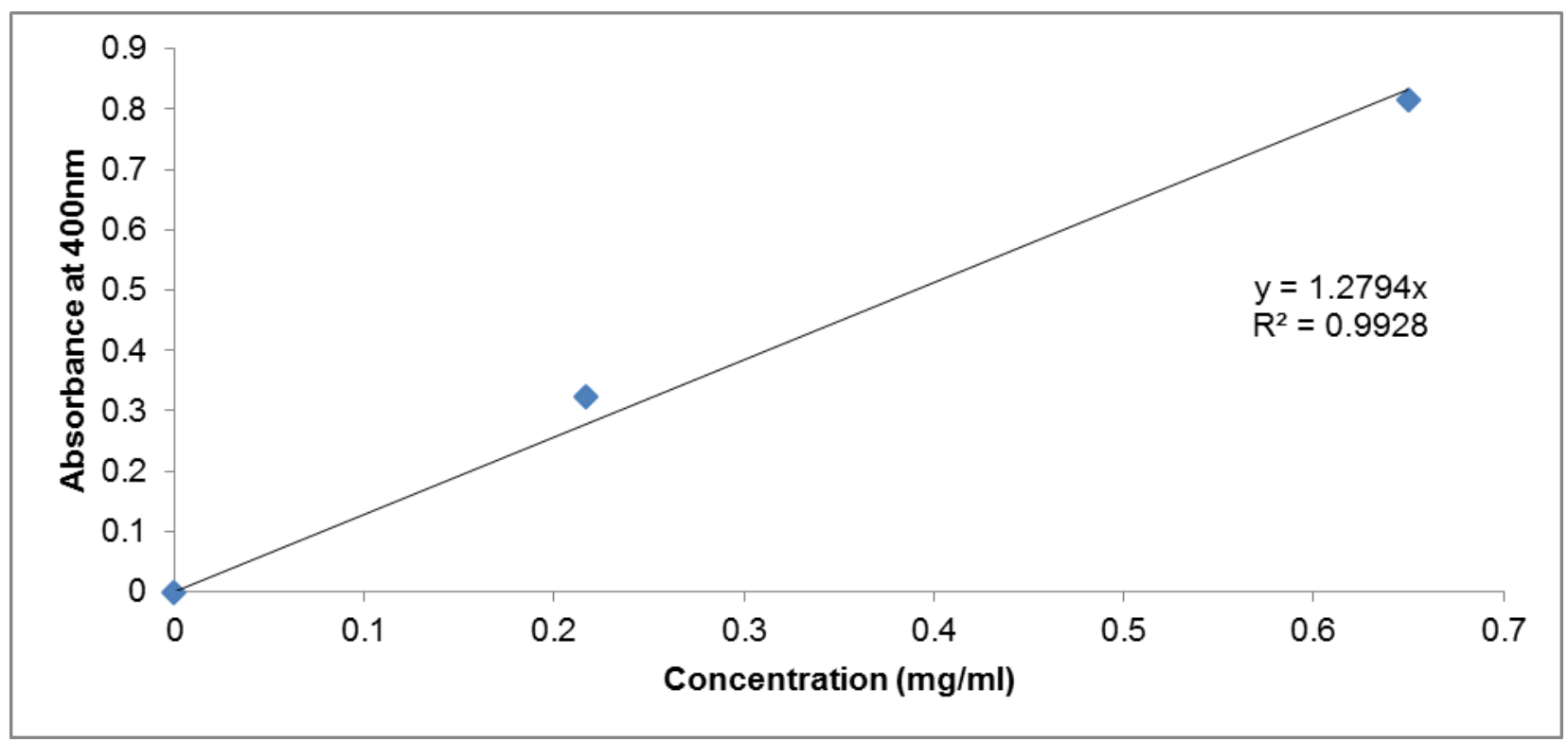

Figure S1: The relationship between absorbance (measured at $400 \mathrm{~nm}$ ) and concentration allows us to calculate extinction coefficient 
a
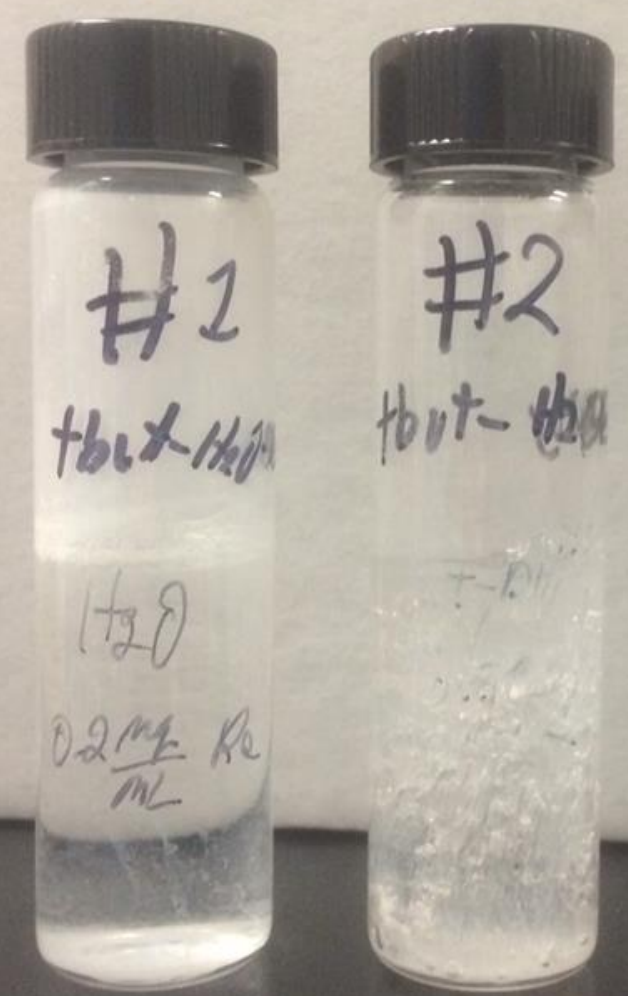

C
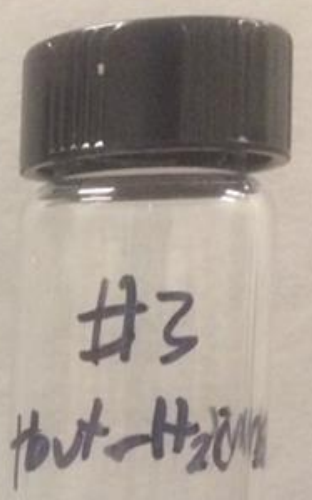

Figure S2: a) Redispersion in pure was not successful as big flakes could be seen floating around and eventually sedimented to the bottom. b) Redispersion in pure t-butanol was challenging because of its freezing point around room temperature. Redispersing in pure t-butanol yielded a "frozen" dispersion; the BNNSs were kinetically trapped after freezing. c) Redispersion in co-solvent was successful as the BNNSs flakes "dissolved" into solvent. 


\section{FTIR-ATR analysis}

FTIR-ATR spectra of pure water and t-butanol were obtained from their respective liquid phases. FTIR-ATR spectra from both vacuum-filtered solvent-exchanged dispersion and vacuum-filtered co-solvent dispersion were obtained from powders that were scraped off from their respective films. Even after vacuum filtering, washing, and drying, the solvent-exchanged sample still displays the characteristic alkane C-H peak $\left(2850-3000 \mathrm{~cm}^{-1}\right)$ and characteristic alcohol OH peak (3200-3500 $\left.\mathrm{cm}^{-1}\right)$, confirming the presence of t-butanol. The samples without any $\mathrm{OH}$ peak were the vacuum-filtered co-solvent BNNS dispersion and the freeze dried BNNSs powder.

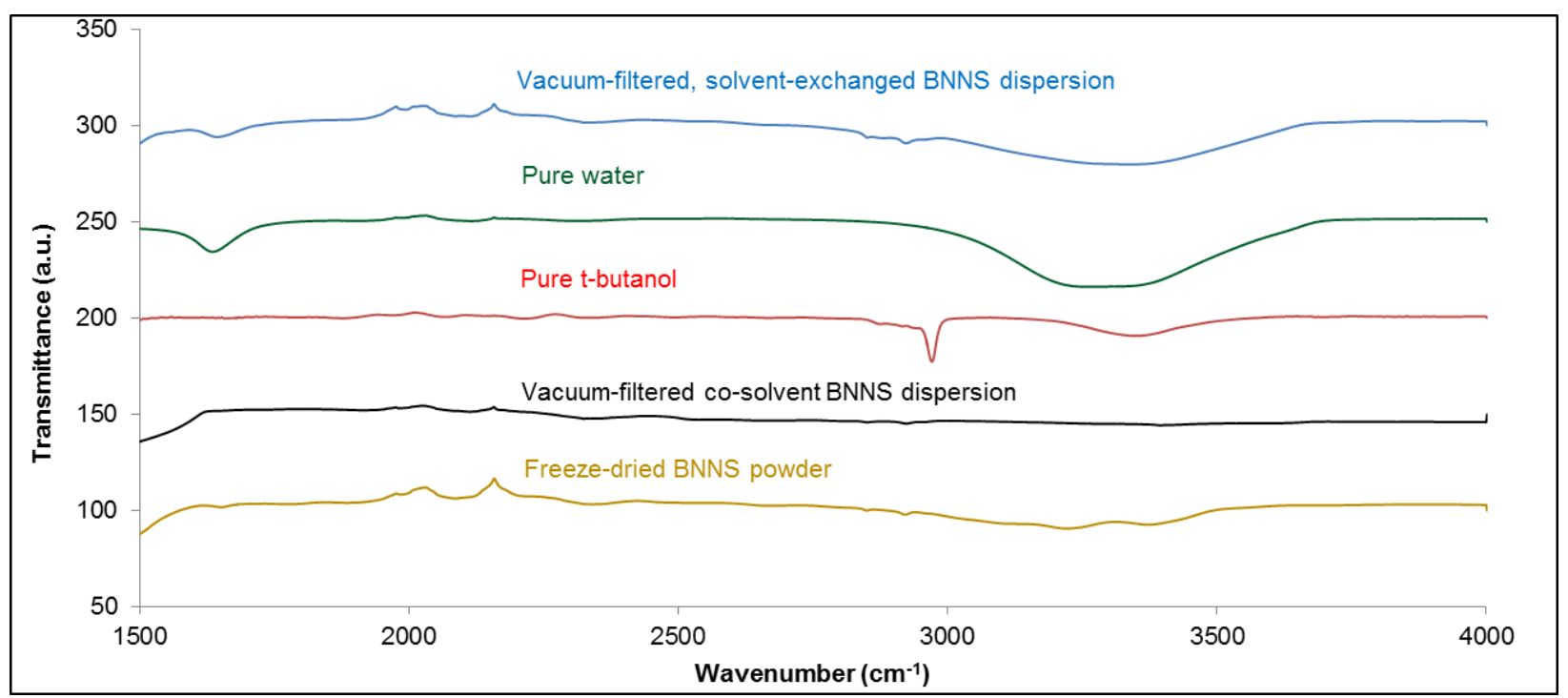

Figure S3: FTIR-ATR of vacuum-filtered, solvent-exchanged dispersion, pure water, pure t-butanol, vacuum filtered co-solvent dispersion, and freeze dried BNNSs powder 

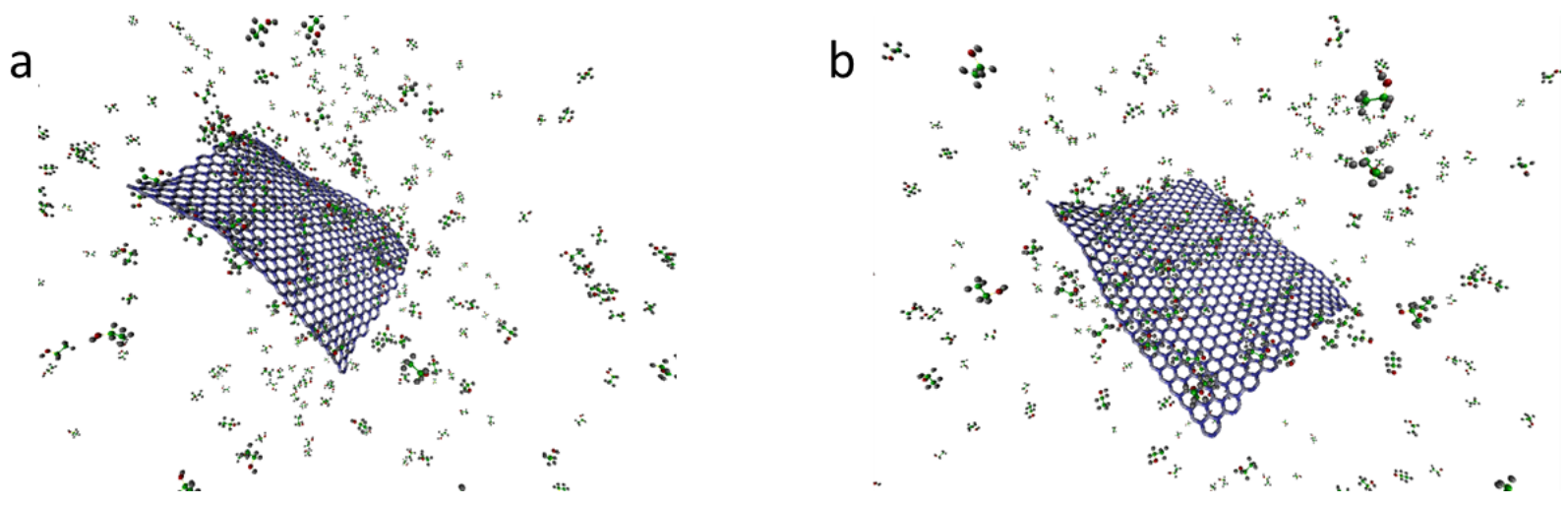

Figure S4: End configurations of two different replica structures for ethanol-BNNS-water system after $8 \mathrm{~ns}$ of production run. Water molecules are not shown for the sake of clarity.
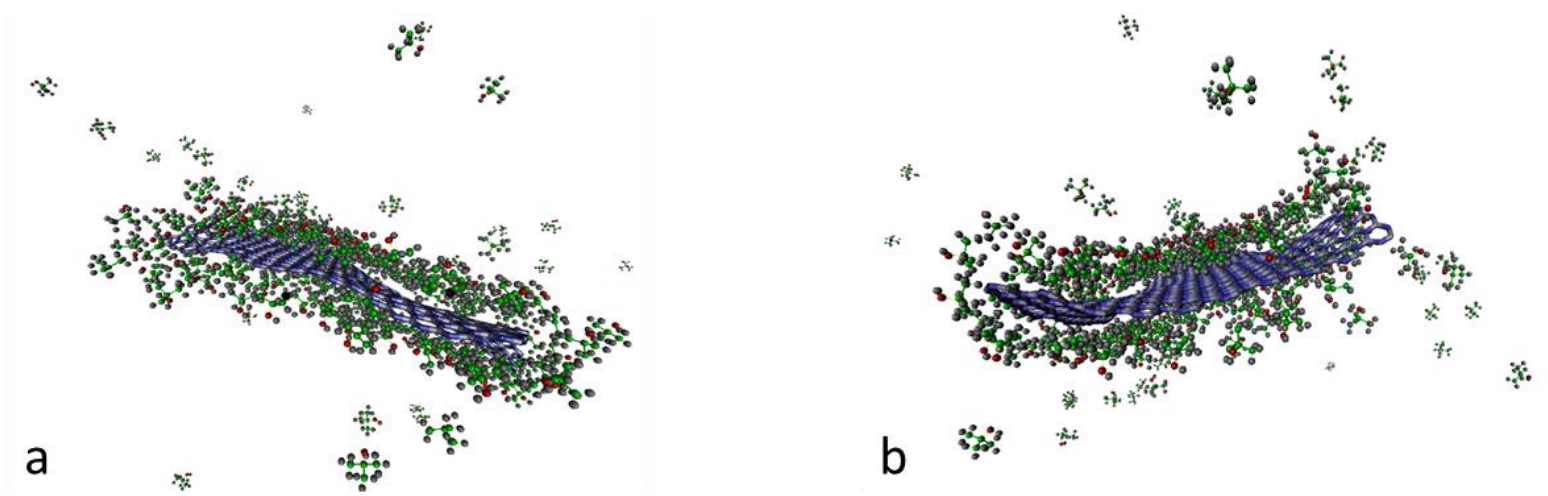

Figure S5: End configurations of two different replica structures for t-butanol-BNNS-water system after $8 \mathrm{~ns}$ of production run. Water molecules are not shown for the sake of clarity.

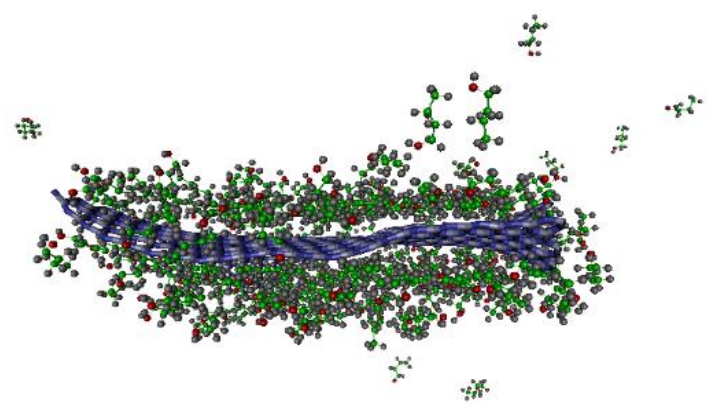

a

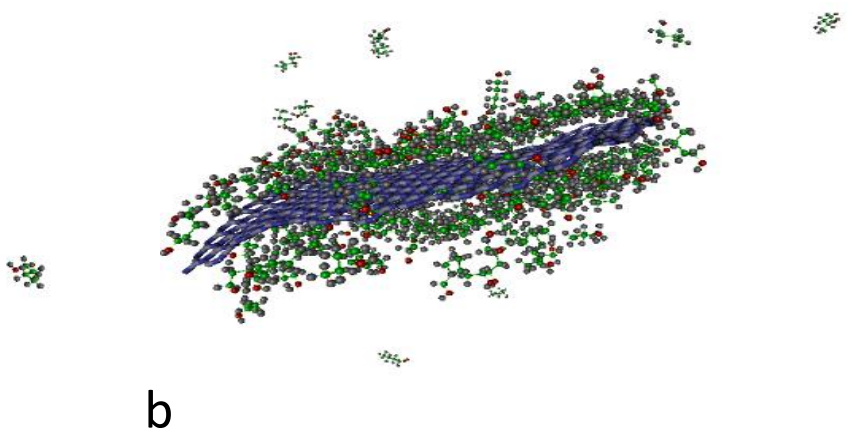

Figure S6: End configurations of two different replica structures for 1-butanol-BNNS-water system after 8 ns of production run. Water molecules are not shown for the sake of clarity. 

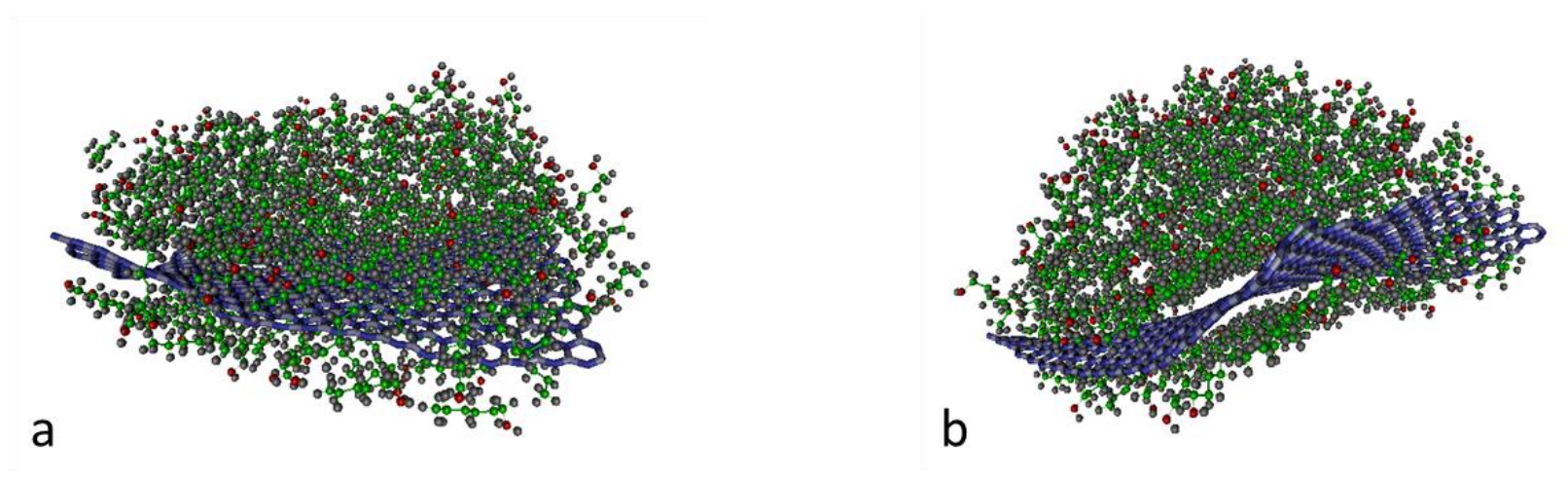

Figure S7: End configurations of two different replica structures for 1-hexanol-BNNS-water system after $8 \mathrm{~ns}$ of production run. Water molecules are not shown for the sake of clarity.
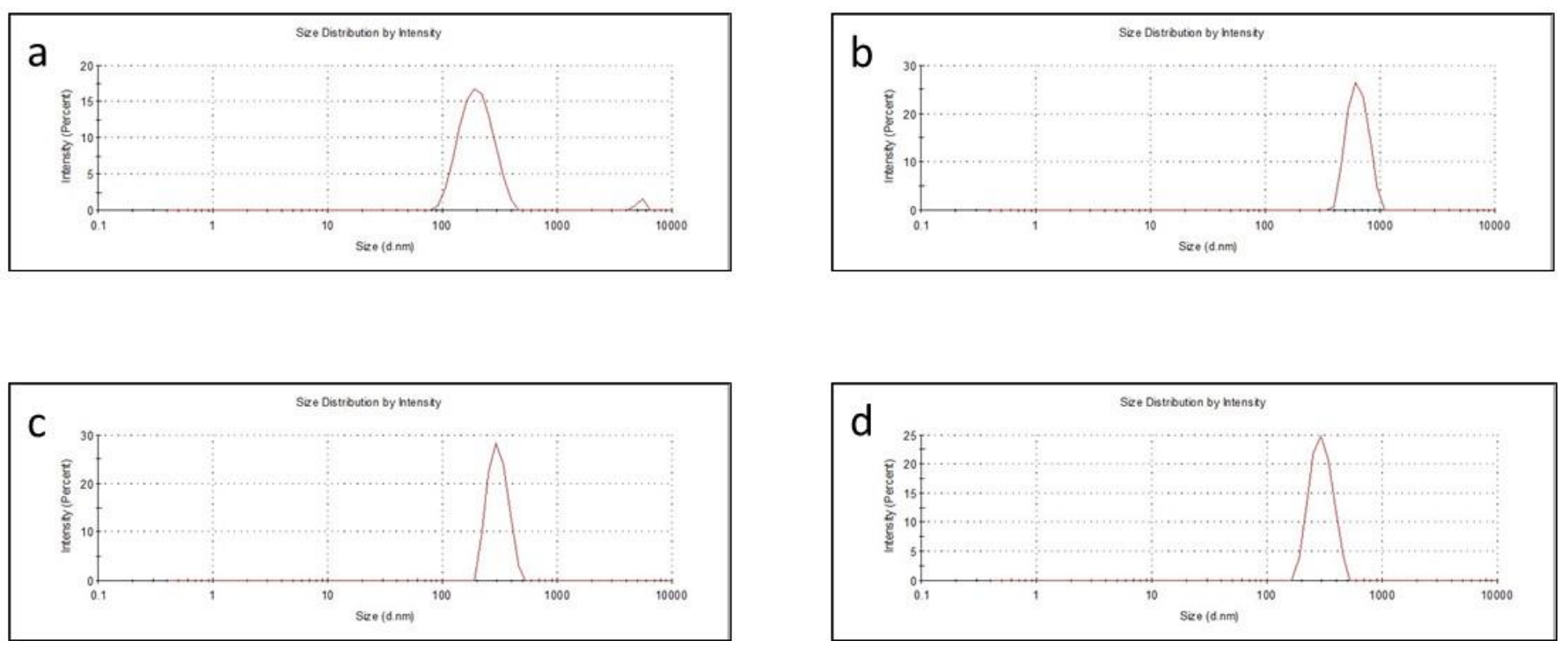

Figure S8: Dynamic light scattering data of various dispersions. a) Size distribution of H2O-BNNS dispersion. b) Size distribution of co-solvent dispersion. c) Size distribution of solvent exchanged dispersion. d) Size distribution of solvent exchanged + PVP dispersion. 